in vivo $32: 1289-1300(2018)$

doi:10.21873/invivo.11379

Review

\title{
Treatment of Articular Cartilage Defects: Focus on Tissue Engineering
}

\author{
BEATA ŻYLIŃSKA ${ }^{1}$, PIOTR SILMANOWICZ ${ }^{1}$, ALEKSANDRA SOBCZYŃSKA-RAK ${ }^{1}$, \\ ŁUKASZ JAROSZ ${ }^{2}$ and TOMASZ SZPONDER ${ }^{1}$ \\ ${ }^{1}$ Department and Clinic of Animal Surgery, Faculty of Veterinary Medicine, \\ University of Life Sciences in Lublin, Lublin, Poland; \\ ${ }^{2}$ Department of Epizootiology and Clinic of Infectious Diseases, \\ University of Life Sciences in Lublin, Lublin, Poland
}

\begin{abstract}
The treatment of articular cartilage defects seems to be one of the greatest challenges in modern orthopaedics. From a medical point of view there are 3 main goals to achieve for cartilage trauma treatment: restoration of the joints motion, pain relief and elimination/delay of the onset of osteoarthritis. Treatment can be divided into conservative (including pharmacotherapy, arthrocentesis and physiotherapy) and surgical. The last comprises reparative techniques, regenerative methods and symptomatic treatment. While both are focused on reconstruction of the damaged cartilage, the difference lies in the type of filling tissue. Reparative techniques include: drilling of the subchondral bone, spongiolisation, abrasion, mictrofracture, and autologous matrix induced chondrogenesis (AMIC). Regenerative methods contain: periosteal and perichondral grafts, mosaicplasty, autologous chondrocyte implantation and matrix-induced autologous chondrocyte implantation (MACI). Nowadays tissue engineering, including gene therapy, is emerging as one of the key approaches to cartilage treatment and holds promises for new achievements and better outcomes of many cartilage diseases and traumas.
\end{abstract}

The treatment of articular cartilage defects in one of the greatest challenges in modern orthopaedics (1-5). Mankind

This article is freely accessible online.

Correspondence to: Piotr Silmanowicz, Department and Clinic of Animal Surgery, Faculty of Veterinary Medicine, University of Life Sciences in Lublin, 30 Głęboka Str., 20-612 Lublin, Poland. Tel: +48 501844978, e-mail: piotrsil@ poczta.onet.pl

Key Words: Animal, cartilage, treatment, regeneration, biomaterial, tissue engineering, review. has struggled with cartilage trauma and its treatment for centuries. As famously observed by the Scottish anatomist and surgeon William Hunter in 1743, cartilage trauma had been recognized as particularly troublesome since the days of Hippocrates because once damaged, it will not regenerate (6-9). A hundred years later, another scholar named Leid made a nearly identical comment, saying that "damaged cartilage fragments never form union, cartilage tissue has no regenerative capacity". Improbable though it may seem, even today, in the age of advanced tissue engineering and regenerative medicine, the above statement still holds true. Despite the undeniable advances in the treatment of chondral trauma, to date, the complete restoration of the physiological condition of articular cartilage still seems to elude us (10-11).

\section{Interspecific Differences in the Macro and Microscopic Structure of Articular Cartilage}

Experimental animal models are the primary choice of research aimed at assessing the effectiveness and safety of new biomaterial solutions. For instance, implants intended for use in the human knee joint are tested in small and medium-sized mammals. So far, literature offers few studies providing a quantitative and systematic comparison between the anatomies of intra-articular structures in various species of animals and humans (12). Although the macroscopic lamellar structure of the articular cartilage is common to all mammal species, certain species-specific differences do nonetheless exist. These should be considered when designing experimental models focused on the treatment of chondral tissue trauma in humans (6). The differences include the varying total thickness of the cartilage in the respective species and the relative height of its respective layers in equivalent joints. 
In humans, most of the research conducted to date has been focused on knees and hips. It is estimated that in an average, healthy, young subject, the typical height of the cartilage covering the medial femoral condyle is between $2 \mathrm{~mm}$ and $4 \mathrm{~mm}$, but can reach up to $6 \mathrm{~mm}$. In experimental animals such as rabbits, sheep or goats the thickness of the equivalent cartilage is $0.25 \mathrm{~mm}, 0.4 \mathrm{~mm}$, and $0.9 \mathrm{~mm}$ respectively. In large mammals such as cattle or horses, cartilage thickness is also roughly within this range. On the other hand, the layers of calcified cartilage and subchondral plate in these animals are considerably thicker than in humans (6).

Significant discrepancies can also be observed in terms of the chondrocyte content per a unit of volume. In humans, cartilage stands out among other tissues due to its very low concentration of cells. The mean content of chondrocytes in the cartilage of the medial femoral condyle is only $0.65 \%$ of the cartilage mass with the values decreasing as we move away from the joint surface. Between the superficial layer and the radiant layer, the value decreases from $2.6 \%$ to $1.2 \%$. Such low values have not been observed in other species. By comparison, in rabbits and goats the density of chondrocytes in the equivalent cartilage is $12 \%$. The distribution of cells has a direct impact on chondral metabolism. Low cell density means that chondrocytes are scattered relatively far from each other. Therefore, a single cell of human cartilage corresponds to a far greater area of matrix than in other animal species. It has been calculated that a single chondrocyte in human medial femoral condyle corresponds to $104,040 \mathrm{~mm}^{3}$ of the matrix. The value is further increased to $106,707 \mathrm{~mm}^{3}$ in the deeper part of the radiant layer. In the equivalent rabbit cartilage these values are approximately ten times lower $(2,13)$.

Discovering the exact three-dimensional structure of the articular cartilage constitutes the starting point in the effort to understand its chemical, physical, and biomechanical characteristics, which determine its physiological functions. The knowledge is necessary in order to determine the correct direction of development with regard to the treatment of chondral injuries. Given the limited ability of articular cartilage to heal itself, clinical intervention is necessary to prevent further damage and limit the extent of osteoarthritis (14).

\section{Research Models of Chondral Healing Processes}

In studies devoted to the healing processes in articular cartilage tissue, rabbits are the most commonly used animals. The size of the femoral condyle and trochlea in these animals is large enough to easily accommodate artificially introduced osteochondral lesions of 3-4 mm in diameter (15). However, the difficultly in applying results obtained from such studies to humans stems from the different potential of myeloid cells. In rabbits repair of osteochondral lesions takes place almost exclusively through proliferation and differentiation of mesenchymal stem cells, without the participation of adjacent healthy cartilage cells. Without intervention, human cartilage shows no or very little capacity for spontaneous healing. Furthermore, the articular cartilage in rabbits is very thin, which means that approx. Eighty percent of the created injury in fact affects the subchondral bone rather than the cartilage itself (15).

From the clinical perspective, three primary goals of cartilage trauma treatment can be identified:

- restoration of the joint's functional capacity by reconstructing the continuity of articular surfaces,

- reduction of pain,

- delay or prevention of the onset of osteoarthritis $(6,16)$.

The unique structural characteristics and not fully understood trauma response of cartilage results in a somewhat non-uniform approach that physicians adopt when treating such injuries (17). The method of treatment depends on: the size, location and depth of the lesion as well as the coexistence of other joint conditions (17). It can be generally divided into conservative treatment and surgical treatment.

Conservative treatment entails pharmacotherapy, various minimally-invasive surgical methods - immobilization, joint puncture, physiotherapy and reduction of body mass.

\section{Pharmacotherapy}

The concept of "slow acting drugs in osteoarthritis, SADOA" has been used in the context of osteoarthritis pharmacotherapy (18). The medicines are roughly divided into two groups:

- symptomatic slow acting drugs in osteoarthritis, SYSADOA; and

- disease modifying osteoarthritis drugs, DMOAD, also known as chondroprotectors or nutraceuticals.

SYSADOA drugs are primarily analgesics. They minimize or eliminate pain and reduce reflex muscle tone. They include two types of substances: steroidal and non-steroidal anti-inflammatory drugs (NSAIDs). Whereas substances qualified as DMOADs are administered to aid the reconstruction of affected intercellular substance in the articular cartilage and prevent the effects of enzymes degrading the matrix. In doing so, the drugs also reduce the severity of clinical conditions and pain. Thus, DMOADs share some of their effects with SYSADOAs and it is notoriously difficult to determine the extent to which their action is purely symptomatic and to which they actually inhibit the disease process (18). Examples of DMOADs include e.g. manganese citrate, pentosane polysulphate, scavengers of free radicals such as: DMSO, superoxide dismutase (SOD), bioflavonoids, methylsulphonylmethane, and omega-3 fatty acids $(18,19)$. It should be mentioned that 
according to North American Nutraceutical Council, chondroprotectors (nutraceuticals) are non-drug substances produced in a purified form or as extracts, administered orally, and containing ingredients facilitating the structural and functional health of the organism. They are regulated by less strict pharmaceutical regulations compared to drugs and are mostly sold over the counter $(18,19)$.

Good examples of drugs qualified simultaneously as SYSADOA and DMOAD are glucosamine sulphate and chondroitin sulphate. They are administered orally and show very good assimilability. Studies on glucosamine conducted on animal models as well as clinical trials in humans, have been conducted since 1994. They revealed that glucosamine sulphate is an efficient analgesic, whose pain-relieving effects last far longer than those of non-steroidal drugs. Furthermore, it helps to inhibit the development of degenerative disease, as measured radiologically with regard to its effect of slowing-down the narrowing of the articular space in human knee joints $(8,18)$.

\section{Minimally-invasive Surgical Methods}

Articular puncture (arthrocentesis) is performed to evacuate excess synovia produced by the synovial membrane as a response to the injuring factor or to inject certain preparations by way of so-called viscosupplementation. In the course of the latter, hyaluronan or sodium salts are introduced intraarticularly to supplement and improve the natural qualities of hyaluronic acid present in the articular fluid, which is responsible for tribological qualities (viscosity and elasticity) of synovia (19). The injection of viscosupplements is intended as a way of increasing the viscosity of synovia, which in turn ensures more effective distribution of articular cartilage nutrients and improves articular motor activity (1819). However, literature lacks specific guidelines in terms of the optimum number of injections, dosage, and the most effective make-up of the administered preparation. It is believed that intra-articular administration of hyaluronic acid is more effective as an analgesic than non-steroidal antiinflammatory drugs or glucocorticoids $(18,19)$.

Other modern therapies include injection of platelet growth factor in the form of platelet rich plasma (PRP) and IL-1 receptor antagonist protein IRAP/Orthokine ${ }^{\circledR}$ therapy (18). These methods can yield symptomatic improvement and temporary reduction of pain. However, to date, there has been no evidence to suggest that any of these techniques can actually contribute to structural reconstruction of a chondral defect $(9,20)$.

The PRP method entails the use of autogenic platelet mass. Platelet concentrate is obtained and concentrated by centrifuging the patient's own whole blood. Fibrin present in the plasma and adhesive particles responsible for cell adhesion serve as carriers for the platelets (21). Platelets actively participate in the formation of clots and most healing processes in the body. They are particularly rich in growth factors and cytokines, which positively influence the proliferation and differentiation of various cell types (20). They are released from platelet $\alpha$-granules under the influence of thrombin, collagen or mechanical factors. Since the 1970s, platelet rich plasma has been used in bone, ligament and tendon tissue engineering (20). Studies pertaining to its applicability to articular cartilage are still at their preliminary stages. In 2010, the first research results on the use of PRP administered intra-articularly to patients with knee joint cartilage defects were published. However, the value of the study is limited by the short observation period, absence of control, and non-normalised method of obtaining plasma $(18,20,22)$.

Orthokine ${ }^{\circledR} /$ IRAP $^{\circledR}$ therapy entails the preparation of the patient's own blood serum, in the course of which the antagonist of the receptor of pro-inflammatory interleukin 1 (IL-1Ra) and growth factors are separated and concentrated $(18,23)$. Such serum is referred to as Autologous Conditioned Serum (ACS). In human medicine, the system is known as Orthokine ${ }^{\circledR}$ therapy and has been in use since 1998. In equine and canine veterinary medicine, it is referred to as $\operatorname{IRAP}^{\circledR}(18,22)$.

The symptomatic treatment entails intra-articular lavage, surgical debridement and curettage, and surgical removal of the affected cartilage (chondrectomy). In human and veterinary medicine, these measures are performed either arthroscopically or by way of arthrotomy. These are the methods of choice in cases of minor cartilage defects. Debridement involves excision of the frayed cartilage, removal of its unstable fragments, evening out the edges of the lesion up to the border of healthy tissue and curetting any potential osteophytes (18). The method was developed by Magnusson over 60 years ago. Joint lavage using a $0.9 \%$ isotonic salt solution facilitates the removal of proinflammatory factors and degenerated dead cells from the articular cavity. It has been suggested that lavage allows the release of surface proteoglycans which can promote later adhesion of reparative cells from the synovial membrane (18). Long-term observation of patients after the use of the aforementioned methods confirmed their beneficial effects. At 14 months follow up, improvement was observed in $74 \%$ of 78 patients. Furthermore, in the course of a four-year follow up of 109 patients, positive results were reported in $63 \%$ and poor results in $37 \%$ of the patients (24).

Very important factors affecting the restoration of articular function in both human patients and animal models are the physiotherapeutic and rehabilitative procedures, including: laser therapy, magnetic field therapy, shock wave therapy, massage and balneotherapy. Non-invasive treatment should be supplemented by efforts aimed at restoring the correct body mass to facilitate proper joint mobility (18). 


\section{Surgery}

Surgical treatment methods include: reparative techniques, regenerative techniques, and symptomatic (palliative) treatment.

Both the reparative and regenerative methods are primarily focused on reconstructing the damaged articular cartilage. Repair of a defect entails filling with tissue of different histology than that of the healthy chondral tissue. Practically, the same equates to recreating tissue continuity but without restoring their original differentiation and full functional capacity (21).

\section{Reparative Methods}

Reparative techniques entail the recruitment of mesenchymal stem cells by interfering with the continuity of cancellous bone vessels (so-called "marrow stimulation techniques") (25). The stimulated biological response of the organism produces scar tissue referred to as fibrocartilage tissue. Such therapies are used in the treatment of minor $\left(<2 \mathrm{~cm}^{2}\right)$ and medium-sized defects (up to $2.5 \mathrm{~cm}^{2}$ ) depending on the patient's activity patterns and expectations (10).

They include the following techniques: drilling of the subchondral bone, spongiolisation, abrasion, mictrofracture, and AMIC method.

Drilling of the subchondral bone was first performed by Pridi in 1959 in an experimental rabbit model. The drilled holes are filled with a haematoma and then a clot rich in multipotent cells and growth factors is recruited from the marrow (26). As the original framework is reconstructed, fibrocartilage tissue is formed. The drilling additionally reduces intraosseous pressure, which relives pain. The downside of the technique is that the drilling process causes thermal damage to otherwise healthy tissue (25).

In the 1970s, Ficat et al. developed a radical technique referred to as spongiolisation. It entails the removal of the whole affected cartilage together with the adjacent subchondral bone $(10,26)$.

In the $1980 \mathrm{~s}$, Johnson introduced the abrasive technique which involves curettage of 1-3 mm of subchondral bone at the site of the chondral defect, which exposes the vascularised layer. The validity of the method remains controversial, however, due to the considerable damage to subchondral bone plate it entails $(17,26-27)$.

In 1984, Steadman introduced the method of microfractures which is now one of the most commonly recommended reparative techniques. It involves the introduction of microholes into the subchondral bone using surgical tools similar to awls, spaced at 3-4 millimetres. An important element of the technique is proper prior preparation of the damaged area. The layer of calcified cartilage needs to be removed from the bottom of the lesion and its edges need to be evened out. The correctly prepared bed will be able to accommodate and retain the forming clot (26). The procedure does not generate excessive heat or entail damage to the subchondral bone plate, as is the case with drilling or abrasion $(9,17,24)$. The procedure is single-stage and relatively simple technically (14). Steadman reported significant improvement relative to the preoperative condition in $80 \%$ of 72 patients undergoing microfracture treatment. However, he accounted only for the patients' subjective opinions, rather than histological data (9). Histological studies conducted on animal models where the aforementioned technique was applied revealed that fibrous tissue containing primarily type I collagen is formed at the site of the defect. However, after 18 months, the quality of this tissue was observed to have deteriorated significantly (14).

The AMIC method (Autologous Matrix Induced Chondrogenesis) is a combination of the microfractures technique and the use of a Chondro-Gide ${ }^{\circledR}$ collagen membrane (Geistlish Biomaterials) $(25,28)$. It was first presented by Behrens in 2003 as a method of improving the effectiveness of the microfractures technique to allow its applicability to more extensive cartilage defects (up to $6 \mathrm{~cm}^{2}$ ). The membrane covers the site of the defect to stabilise and protect the forming clot, stimulate production of type II collagen, and reduce bleeding into the articular cavity. Its three-dimensional structure allows it to properly direct the forming collagen fibres. It is held in place by tissue adhesive or sutures. The commercially available Chodnro-Gide collagen membrane is manufactured from type I and III collagen fibres from swine tissues $(17,25)$.

In the course of long-term research and observations of patients undergoing reparative treatment conducted by Steadman, among others, the clinical effectiveness of this approach has been generally assessed as satisfactory (8). Analyses of the makeup of the reconstructed tissue revealed that it primarily consists of fibrocartilaginous tissue. Unfortunately, due to the insufficiency of structural and mechanical qualities of fibrochondral tissue, the long-term efficacy of the aforementioned methods is somewhat debatable. It is generally accepted that treatment entailing the use of marrow stimulation techniques can provide short-term symptomatic relief $(27,29)$.

\section{Regenerative Methods}

Tissue regeneration includes replacement of damaged cells and intercellular substance while preserving their microarchitecture and biomechanical functions.

Regenerative methods include:

- periosteal and perichondral grafts,

- autologous and allogenic osteochondral grafts (mosaicplasty, OAT - Osteochondral Autologous Transplantation; Osteochondral Autograft Transfer).

- Autologous Chondrocyte Implantation (ACI) - (synonymous to ACT: Autologous Chondrocyte Transplantation).

- Matrix-Induced Autologous Chondrocyte Implantation (MACI). 
- treatment of chondral defects using tissue engineering methods and biomaterials.

\section{Periosteal and Perichondral Grafts}

The procedure entails covering the area of the cartilage defect with a flap of periosteal or perichondral tissue (30). The earliest reports on its use date back to the 1980s. A modification of this method entails the introduction of bone marrow under the grafted flap (17). The inner layer of periosteum is known as the growth layer (cambium) and is rich in blood vessels and progenitor cells with a high mitotic potential: osteogenic and chondrogenic (29, 30). The expression of a given phenotype depends on a number of factors. The formation of chondroblasts is influenced by, e.g.: low oxygen pressure, limited availability of nutrients and the presence of other cells (29). Literature confirms the effectiveness of using periosteal tissue in the treatment of chondral defects based on 3 studies conducted in different periods and involving 24 patients. In none of the studies were biopsies taken to provide material for histological and biochemical assessment of the newly formed tissue. Only one of these entailed a macroscopic assessment of the healed injury after 12 months. It was reported that the tissue was similar to healthy cartilage (29). In all clinical trials the effectiveness of the procedure was assessed based on the patients' subjective opinions with regard to the joint's functional capacity and the experienced discomfort (29). The use of perichondral tissue, which also contains mesenchymal cells, remains controversial because articular cartilage, being one of the organism's hyaline cartilages, is not in fact covered with perichondrium. Perichondral grafts have been used in several experimental animal trials and clinical trials in humans. The material was harvested from rib cartilage. In the longest observation period of 52 months, satisfactory results were reported in $38 \%$ of the cases. Due to the reported success rates the two methods are currently only rarely used in medical practice.

\section{Autologous and Allogenic Osteochondral Grafts}

Osteochondral grafts are cartilage fragments with the adjacent subchondral bone, usually cylindrical. The earliest reports on their use can be traced back to 1908 and 1925 (26). The method found use in the treatment of mediumsized defects - up to approximately $3-4 \mathrm{~cm}^{2}$.

Two methods using autological grafts can be distinguished: Mosaicplasty and osteochondral autologous transplantation (OAT). Both were introduced in the late 1990s by Hangody, Bobic and Outerbridge. The differences between the two are mainly found in the size and number of cylinders collected, and consequently the method of filling the defect (26). Mosaicplasty entails selecting a number of cylindrical osteochondral fragments of several millimetres in diameter, and subsequently using them to fill the lesion in a way reminiscent of mosaic tiles. In the osteochondral autologous transplantation (OAT) technique, the collected cylinders are significantly larger, up to $9 \mathrm{~mm}$ in diameter, so only one or two of those are sufficient for a typical defect. By carefully adapting the number and diameter of grafts to be used, even $90 \%$ coverage of the affected area can be ensured (20). The potential benefits of this method include a potential for nearly ideal reconstruction of the cartilage surface and the layer of subchondral bone, filling the defect with actual articular cartilage and therefore recreating the natural barrier between synovia and subchondral bone (31). Grafts are stabilised at the site of the defect by way of manual press-fit (9). No additional attachment methods are required (30). The procedure is completed in a single stage, which reduces the risk of graft rejection. However, the collection of graft tissue is invasive, and the most common complications occur at the donor site, including haematomas, inflammations, deterioration of articular function (so-called "donor site morbidity") $(9,26)$. Due to the above, the applicability of the method is reduced by the limited availability of articular surfaces which can serve as sources of osteochondral cylinders, consequently it is not sufficient for the treatment of extensive joint damage. It can be applied only to weakly encumbered places with a sufficient thickness of articular cartilage tissue (30). If the cylinders are too short, they cannot be effectively anchored at the bottom of the defect. In time, the empty space between the cylinders is filled with fibrous tissue, which contributes to a reduction of joint congruence and progressive degenerative changes (910, 20). Literature confirms superior effectiveness of the OAT method, as compared to microfractures, in the treatment of knee joint cartilage trauma in professional athletes (32). The only household animal disease in whose treatment attempts have been made to apply autologous osteochondral grafts is osteochondrosis (31). This type of surgery has been attempted in dogs suffering from osteochondrosis of the knee joint and the medial humeral condyle, and horses with cysts on the femoral condyle subchondral bone and distal metatarsal and metacarpal epiphysis $(31,33)$. The recurrent issue is the traumatisation of the osteochondral cylinder donor site as the only available source of the same is the knee joint which also happens to be the most likely site of cartilage injury. In their study on the effectiveness of the OAT procedure in the treatment of cubital joint osteochondrosis in dogs, Fitzpatrick et al. reported significantly varied post-surgery results (31). However, the development of osteophytes in the operated joints was observed in all dogs. Moreover, the experiment involved relatively short observation periods (31).

Another method involves the application of autogenous, crumbled (spongiolised) osteochondral grafts. The approach 
was introduced by Stone and Wigenblach (17). The defect cavity undergoes curettage with intentional blood vessel damage. The collected osteochondral blocks are crumbled and introduced in the form of homogenous mass into the bleeding defect site. Stabilization is reinforced with a fragment of the joint's synovial membrane $(17,34)$.

Allogenous grafts may be fresh, not older than 7 days, or frozen, collected from another donor. An allogenic osteochondral transplant was first performed in 1975 by Allan Gross to fill a large articular defect due to the en bloc resection of a tumour $(26,35)$. This technique is used primarily in the treatment of large chondral defects. Articular cartilage, being deprived of blood vessels, is unable to trigger a reaction from the immune system. However, negative immunological response of the grafted, vascularised subchondral layer remains a potential risk factor (29). Another downside of this technique is the potential danger of transmitting communicable diseases $(9,20)$. In 1987, Stevenson et al. tested the effectiveness of two types of allogenic grafts: fresh and frozen, in the course of experimental treatment of osteochondral defects in dogs (29). Freezing was found to reduce the immunogenicity of foreign tissue, but at the same time reduced the survival rate of chondrocytes (29).

\section{Autologous Chondrocyte Implantation (ACI) Method}

The ACI method (Autologous Chondrocyte Implantation) was first proposed by Brittberg in 1994 (36). It is the only method of reparative articular cartilage treatment to have been officially approved by the American Food and Drug Administration (FDA) (36). It is mainly used in the treatments of large, grade 3 and 4 chondral defects, up to 10 $\mathrm{cm} 2$, where other methods are not viable options $(28,37$, 38). The procedure involves two stages. Firstly, a fragment of healthy cartilage (bioptate) is collected from the patient and passed on to the laboratory where a chondrocyte culture is started. It is generally agreed that the mass of the bioptate should be between 200 and $300 \mathrm{mg}$, which corresponds to approximately 300,000 cells (27). Chondrocytes are cultured in adequate conditions for 2-3 weeks, which is enough to increase their number by around thirty times $(9,17)$. The second stage takes place between 6 weeks and 18 months from the first, although at times a hiatus of even several years is possible (9). Similarly to other techniques, the bottom of the defect cavity is cleared and levelled. An important factor influencing the success rate of the procedure is the correct height of the adjacent healthy cartilage as the same is used for the purposes of stabilizing sutures. A periosteal flap should be attached around the area of the defect. A chondrocyte suspension is then injected into the thus sealed cavity. One should make sure that the cells are distributed evenly. Additional reinforcement can be provided by adding a layer of fibrin sealant. ACI is among the most costly and time-consuming methods due to its two-stage procedure. Another downside is the need to perform a biopsy, which entails the risk of damage to the donor site and hindering joint mobility. Another potential problem may be related to excessive proliferation of cells from the periosteal flap, leading to osseous overgrowth of the defect cavity, which is observed in $5-25 \%$ of the cases $(20,29)$. Furthermore, the use of sutures can lead to local irritation and damage to the surrounding healthy cartilage (27).

\section{The Matrix-induced Autologous Chondrocyte Implantation (MACI) Method}

It is a variation of the technique described above $(26,28$, 39). Instead of periosteal tissue, it employs a bilayer membrane of purified bovine type I collagen fibres (commercially available medicinal product MACI ${ }^{\circledR}$, Verigen Transplantation Service, Denmark). It is coated with a suspension of the patient's own previously collected and cultured chondrocytes concentrated at 1 million cells per $\mathrm{cm}^{2}$. This technique ensures even distribution of the cells. Implantation of the membrane requires only the use of a tissue adhesive and replacement of the periosteal flap eliminates the risk of callus. As declared by the manufacturer, in between 1 in 10 and 1 in 1,000 treated with the MACI product, there is a risk of excessive chondral tissue growth and graft detachment. Other potential complications are related to the surgical procedure itself and include the risk of infection, haemarthrosis, and haematoma (39). Bartlett et al. published the results of randomly selected clinical trials in 91 patients treated with the ACI or MACI method, a year after the surgery. They observed no statistically significant differences between the two methods in terms of the functional capacity of the joint or the macroscopic image of the newly formed tissue (29).

In cases involving very extensive damage to the articular cartilage as well as periarticular degenerative lesions, the last available resort is alloplasty (endoprosthetics) of the affected joint $(40,41)$. Despite continued efforts aimed at improving the composition of implants, the incidence of complications after endoprosthetic surgery in humans remains high. In the case of total hip replacement, it can be as high as $70 \%$ within 10 years from the surgery, and in the case of shoulder joint replacement it affects $14 \%$ of patients (41).

The continuous development of the treatment techniques described above resulted in a significant improvement in terms of their clinical effectiveness. However, the quality of the produced reparative tissue continues to present problems. So far, none of the above methods has been able to produce durable hyaline cartilage capable of withstanding significant mechanical strain in the long-term $(4,7,20)$. It seems that 
the future of chondral trauma treatment lies, similarly to so many other fields of modern medicine, in tissue engineering and gene therapy $(2,17)$.

\section{Treatment of Chondral Defects with the Use of Tissue Engineering}

Tissue engineering was emerged as an independent scientific field in 1988, when it was discussed by doctor Fung from the University of California during a conference of the US National Science Foundation (42). It continues to develop as an interdisciplinary field combining medical knowledge related to surgery, physiology, cell biology and molecular biology with the methods of biotechnology and material engineering, including polymer chemistry. It aims to develop biological substitutes capable of restoring, maintaining or improving tissue or organ functions $(17,21)$. Tissue engineering focuses around 3 main research directions: the search for the adequate source of cells for the purposes of potential tissue regeneration, the development of suitable media (scaffolds, matrixes), and the attempts to influence cell proliferation and differentiation through the application of various regulating factors and bioreactors. The above "triad" of tissue engineering was first proposed by Marx and then reiterated by Lynch in the 1990s $(21,30)$.

\section{Sources of Cells for Potential Tissue Regeneration}

Chondrocytes seem to be the most obvious type of cells to be used in the treatment of chondral defects. However, experience provided by the application of the ACI technique revealed a number of problems with this choice. Chief among those is the fact that mature chondrocytes tend to change their phenotype while cultured in laboratory conditions. To prevent this, one has to adhere to a very strict procedure and not maintain a culture for more than 4-6 weeks (27). Chondrocytes most often transform into cells similar to fibroblasts, which entails a change in the constituents of extracellular matrix they produce $(2,7,30)$. The conducted experiments revealed that the introduction of growth factors such as TGF- $\beta 1$, FGF- 2 , and IGF- 1 can retard the process of differentiation but is nonetheless insufficient to prevent it. Moreover, under in vitro conditions, chondrocytes are prone to age considerably faster (2). The small number of available cartilage cells and their low mitotic activity mean that obtaining large populations from cellular cultures is highly problematic. Cellular culture should be maintained until the density of 1 million chondrocytes per $1 \mathrm{~cm}^{2}$ of the defect is reached. Such number of cells is considered as optimum for implantation. Therefore, research is currently conducted into the possibility of harvesting other potential sources of chondral cells, e.g. conchal cartilage. However, the use of chondrocytes other than articular can lead to other difficulties, such as the need to use appropriate cell differentiation stimulants to allow them to produce the right kind of matrix (17).

Stem cells constitute another pool of undifferentiated cells with high regenerative potential and, at least theoretically, unlimited capacity for division, capable of self-regeneration and transformation into any cell line (43). They are present in the tissues of all multicellular organisms. Depending on their differentiative potential, stem cells can be described as toti-, pluri-, multi-, or unipotent (43). Stem cells are also classified in terms of their origin as embryonic, foetal, or "adult" (somatic). The primordial stem cell is the totipotent zygote. Embryonic stem cells are harvested for research from the blastocyst inner cell mass (44). The risk of neoplasia due to uncontrolled cell division after a transplant (formation of teratomas) as well as ethical concerns are the reasons why studies on embryonic and foetal stem cells are currently still at their preclinical stages. Adult somatic stem cells (ASC Adult Stem Cells) play a key role in maintaining homeostasis and integrity of tissues and organs $(2,18,45)$. They are a group of heterogenic cells with high adhesive capacity. They can be either multipotent or unipotent (46). Under in vitro conditions, they develop forms reminiscent of fibroblast colonies (43). They are capable of expressing a wide array of common surface receptors from CD, STRO, and MSCA groups $(2,43)$. Although they can be harvested from nearly all tissues in the body, their numbers are scarce and their capacity for differentiation is considerably lower when compared to embryonic stem cells. However, due to the absence of moral constrains and the ability to use adult stem cells in autologous grafts this type of stem cells currently enjoys the greatest interest among researchers (43).

In recent years, stem cells have been harvested from a variety of tissues, e.g. bone marrow, adipose tissue, skin, hair follicles, periosteum, skeletal muscles, synovial membrane, and dental pulp $(2,44)$. The most thoroughly investigated types include marrow-derived hematopoietic cells and mesenchymal marrow and adipose stem cells (MSCs) (30, 4748). The chondrogenic potential of marrow-derived hematopoietic stem cells was confirmed in 1998 by Johnstone who introduced TGF- $\beta 1$ to the culture $(2,30)$. The cells started to produce type II and X collagen while the number of type I collagen molecules produced was decreased. In another study, Bosnakowski also demonstrated the ability of stem cells to differentiate into chondrocyte without the introduction of growth factors (2). The downside of this method is its invasiveness as for bone marrow to be harvested the patient needs to undergo general or intraspinal anaesthesia. Moreover, the number of connective-vascular tissue cells that can be obtained through this method is relatively small, only around 1 per 105 (43). As a consequence, it is necessary to multiply the number of cells in an ex vivo culture. This in turn requires the use of appropriate media and growth factors. It also entails 
the risk of varying differentiative capacity in the course of the culture or even loss of the same (43).

In recent years, a popular source of mesenchymal stem cells has been found in adipose tissue $(18,48-49)$. Similarly to bone marrow, adipose tissue is of mesodermal origin and contains a heterogenic population of stem cells known as ASCs (Adipose-derived Stem Cells). As extracted from numerous published papers, the cells maintain their ability to differentiate into osteocytes, chondrocytes and myocytes (48). They also show considerable angiogenic potential (43, 49). However, their greatest advantage is the ubiquity and abundance of adipose in the organism, which means that the harvest procedure is not particularly invasive. Liposuction procedures are well tolerated, and the associated risk of complications is only around $0.1 \%$ (43). Moreover, the method facilitates high survival rate of the harvested cells, even up to $98-100 \%(43,45-46)$. Adipose-derived stem cells are easy to culture outside the organism and, compared to cells obtained from other sources, age at a lower rate. On the other hand, the chondrogenic potential of adipose-derived stem cells is lower than that of marrow-derived cells $(2,36)$. It is important that anoxic conditions be maintained in the culture as only then can HIF 1 (Hipoxia Induced Factor 1) be activated. It inhibits the process of cell differentiation and maintains their non-differentiated state (43). Another problem is the number of cells produced. Stem cells constitute between 1 and $2 \%$ of all nucleated cells isolated from adipose tissue. In order to obtain a pure population of several million stem cells, long-term cultivation under in vitro conditions is required. This significantly extends the duration of clinical treatment. Therefore, a common indirect solution is to transplant the full fraction of nucleated cells containing an unknown percentage of stem cells, referred to as the adipose-derived - stromal-vascular fraction (AD SVF) (18, 46). Furthermore, cell selection is further complicated by the fact that to date, a molecular marker identifying stem cells has not been developed. Therefore, the only method of isolating stem cells from other cells is by way of adhesion to the plastic walls of the container (36). Yet another downside mentioned in literature is related to the low survival rate of cells in the recipient's organism after the graft. A study conducted in horses revealed that 10 days after the procedure, less than $5 \%$ of the transplanted cells survived at the injection site $(45,50)$. Other studies also revealed the risk of neoplastic transformation, steatosis and ectopic mineralisation of the cell suspension after the injection. Moreover, the exact mechanism of stem cell behaviour after transplantation remains a matter of some controversy. In the context of articular cartilage, the latter is in fact one of the most important problems. During the period of skeletal development, stem cells form two types of cartilage, which in turn form the primary and secondary ossification centers. The final stage entails the degeneration and hypertrophy of chondrocytes and their gradual transformation into osteoblasts. This results in calcification of the matrix and formation of bone tissue. Therefore, the greatest challenge in the context of employing MSCs for the purposes of chondral reconstruction is the preservation of their original phenotype and prevention of their transformation into bone progenitor cells. Indeed, to date, the direct transformation of stem cells into the target tissue cells has yet to be confirmed (36). It is now increasingly suggested that stem cells have a paracrine effect, merely facilitating the endogenic reparative process by secreting numerous cytokines and growth factors (18). Several scientific reports are available with regard to the use of marrow-derived stem cells in the treatment of human articular cartilage defects. The therapies utilized a mixture of stem cells suspended in collagen gel, which was introduced into the cleared defect cavity in the cartilage of the medial femoral condyle and patella under the periosteal flap. However, no significant advantages of the procedure, neither histological nor clinical, were observed relative to the control treated without the use of stem cells (36).

In veterinary medicine, stem cell therapy has been attempted in the treatment of tendon trauma in horses and canine degenerative joint disease (18). In the case of the latter, animals qualified for the study were dogs diagnosed with bilateral degenerative and inflammatory osteoarthrosis of the coxa. Treatment entailed intra-articular injections of a suspension of vascular fraction stromal cells derived from adipose tissue. What is rather curious in this context, however, is the fact that despite alleged "thousands" of procedures conducted in USA and GB, only a handful of papers have been published to date with regard to the actual clinical efficacy of the method. Moreover, the results that are presented tend to be inconclusive and largely subjective.

\section{Construction of Media (Scaffolds, Matrixes)}

All sorts of elements coming into contact with tissues of a living organism are produced from biomaterials. In accordance with the findings of the Biomaterials Consensus Conference at the National Institute of Health of 1982, biomaterial is any substance other than a drug or a combination of synthetic or natural substances, which can be used at any time for the purpose of complementing or replacing the tissues of an organ or its part in order to take over their functions $(42,51)$. In 1994, Freed et al. developed the criteria characterizing a perfect biomaterial used in tissue engineering. The primary quality of biomedical materials is their biocompatibility (biotolerance), i.e. ability to harmoniously interact within living matter. The quality is usually defined by non-toxicity, absence of irritative effect on tissues, and lack of immune response to the original material and the products of its gradual degradation $(42,51)$. A medium should provide a temporary scaffold for the newly 
forming tissue and undergo complete resorption once the reconstruction is complete. Furthermore, the degree of the scaffold's degradation should correspond to the rate of tissue reconstruction (52).

Biomaterials are used in the production of various implants employed in virtually every field of medicine. The most commonly used biomaterials include natural and synthetic polymers, ceramic materials, steel and its alloys, composite and carbon materials (51).

Implants used in the reconstruction of articular cartilage are made primarily from natural and artificial polymers, as well as from composite materials. They come in the form of sponges, hydrogels, lattices, membranes, or nanofibers $(2$, 11). The surfaces of biomaterials are typically modified to improve their hydrophilic qualities, porosity and roughness, which facilitate better interaction between the medium and the cells $(53,54)$. The assumption is that the ideal biomaterial should be characterized by adequate micro- and macro-porosity, stability in the tissular environment, biodegradability, and sufficient structural strength to withstand the mechanical stress inherent in the joint's physiological functions $(2,42)$.

Biomaterials made from natural protein (collagen, fibrin, silk fibroin, gelatine) or polysaccharides (alginate, agarose, chitosan, hyaluronic acid) are characterized by good biocompatibility and biodegradability (30). Their surfaces contain ligands recognized by cellular receptors, which facilitates graft adaptation in situ (42). It was demonstrated that culturing chondrocytes on three-dimensional media such as alginate, agarose, and fibrine gel tends to stabilize their phenotype and contribute to increased production of extracellular matrix constituents such as type II collagen and glycosaminoglycans. The medium also influences the chondro-inductive effect towards somatic stem cells (2). The downside of natural media is their low mechanical strength and relatively high degradation rate (11). Due to the content of natural ligands, the rate of degradation in the organism is not uniform and depends on the individual traits of the host (16). Another disadvantage of biopolymers is the risk of negative immunological reaction and transmission of disease.

Currently, the greatest research interest is focused in the context of synthetic biodegradable polymers such as polylactide (PLA; polylactic acid), polyglycolide (PGA; polyglycolic acid), polyethylene glycol (PEG), their copolymers such as PLGA - polylactide/polyglycolide copolymer, polycaprolactone, or poly (propylene fumarate) $(2,4,16,55)$. Synthetic materials are characterized by significant mechanical strength, they are handy and easy to form into any shape needed. They are also immunologically neutral and entail no risk of transmitting pathogens $(30,56)$. Their undeniable advantage stems from the capacity for comprehensive modifications in terms of their chemical and mechanical properties as well as their rate of degradation.
One should also consider the consistency of their production (the ability to ensure that different batches are of the exact same quality) $(4,16,55)$. Resorption in the organism takes place by way of hydrolysis, enzymatic breakdown or phagocytosis and takes between several months and two years $(51,56,42)$. Disadvantages include cellular level interactions and transmission of intercellular signals, which tend to be weaker on synthetic rather than natural media (11). Such materials also carry the risk of inducing a local $\mathrm{pH}$ increase at the implantation site due to the accumulation of polyacid breakdown products (56). In the case of chondrocyte cultures on synthetic media, an increase in proteoglycans production and increased proliferation of chondral cells were observed (2). Currently, the most commonly used synthetic polymers are the polylactic and polyglycolic acids, which are now routinely produced for medical applications (30).

Among newly developed implantation materials, one should take particular note of materials based on calcium phosphates, especially hydroxyapatite bioceramics (HAp). It can be both natural and synthetic. The name "apatite" was coined by Werner in 1790 and stems from the Greek "he apate", which means a deception, a trick (55). Due to its chemical and mineral similarity to the non-organic matter found in bones and teeth, hydroxyapatite does not cause post-implantation cytotoxicity or carcinogenicity. It is characterized by a high level of biocompatibility both with soft and hard tissues. However, the material's most important quality in the context of medical applications is its bioactivity, which is the key to supporting bone regeneration (57-58). Hydroxyapatite is also an osteoinductive and osteoconductive substance with the unique ability to form direct bonds with bone tissue (59).

Composites are bi- or multi-ingredient materials whose qualities are different from those of the respective substances forming them. In the context of articular cartilage engineering, the most promising results seem to be obtained for biomimetic media, i.e. composites combining natural and synthetic components and providing the benefits of both. In recent years, an exponential increase in the interest in nanocomposite materials has been observed. These are combinations wherein one or more elements are considered in the nanometric scale. This allows the composite material to engage in interactions at the atomic and molecular level, which allows it to display certain unique qualities (57).

\section{New Directions in the Treatment of Articular Cartilage Gene therapy}

The concept of gene therapy is to take direct advantage of the DNA material responsible for encoding a given bioactive factor (60-62). Due to the fact that most factors are proteins with short half-life periods, and the thick chondral matrix is a barrier not easily penetrated by protein, a more reliable 
method of their delivery is provided by gene transfer. Selected genetic material is delivered directly to the target cell or at the site of trauma with the use of appropriate vectors: viral or non-viral (liposomes, electroporation process (EP) (62-63). The target cells for genetic vectors may be stem cells, synoviocytes, chondrocytes. There are three possible ways of introducing vectors: injecting the gene vector or genetically modified cells (transgenic) into the articular cavity; ex vivo genetic modification of cells and direct transplantation to the site of chondral trauma by way of arthrotomy (ex vivo approach); delivering the modified vector directly to the site of the defect during arthrotomy (in vivo approach).

One of the first genes induced in vivo was the IL-1 receptor antagonist (IL-1RA). Gene transfer for IL-1RA was used in the treatment of chondral defects in a mouse, a rat, a rabbit, a $\mathrm{dog}$, and a horse. The treatment yielded a reduction in the intensity of the clinical symptoms of degenerative disease as well as lessened cartilage degradation. IL-1RA was also tested in preliminary clinical trials in humans. The gene for IL-1RA was delivered with the use of a retrovirus vector to autological synoviocytes harvested from the metacarpal-digital joints of patients suffering from rheumatoid arthritis. After a 1-week culture, the transgenic cells were injected intra-articularly. A week later the patients underwent joint endoprosthesis implantation. The removed metacarpal-digital joints underwent an analysis which confirmed local expression of IL-1RA and therefore the effectiveness of the method (62-63).

\section{Artificial Articular Cartilage}

Attempts are also made to create artificial chondral matrix to serve as a non-cellular, three-dimensional scaffold intended for the treatment of degenerative diseases. The challenge here is to create a material which would not only recreate the microarchitecture of the respective chondral layers but also have the ability to withstand significant loads and ensure frictionless mobility of articular surfaces. So far, the best results have been reported for hydrogels which constitute a relatively new chapter in the history of tissue engineering. The materials' qualities make them somewhat similar to soft tissues while also providing biocompatibility, thrombogenicity and good permeability for oxygen and other water-soluble metabolites. They form environments which are very effective and conducive to accelerated cell growth when compared to polymeric membranes. The highly hydrated hydrogels mimic the chemical and physical conditions of the extracellular matrix, which facilitates good integration with the site of trauma as well as efficient multiplication and differentiation of cells. The most promising hydrogels to date are those produced on the basis of poly(ethylene glycol), poly(vinyl alcohol), or copolymers of poly(ethylene oxide) and poly(propylene oxide) $(32,40)$.
When considering the contemporarily available methods of articular cartilage trauma and the overall progress made in this respect over the last 20 years, one can conclude that considerable improvement in terms of clinical results has indeed been achieved. The primary problem, however, remains the quality of the articular cartilage produced (17).

As follows from the above literature review, articular cartilage defects continue to pose a pressing therapeutic problem (10). None of the currently available methods can be said not to have any disadvantages, therefore, the search for new therapeutic methods relying on the use of biomaterials can potentially yield considerable benefits in the future. Regardless of the method adopted in the treatment of chondral trauma, one must account for the fact that every joint is just one of the elements of a complex mechanism whose proper function depends on unhindered cooperation between all of its respective parts. Any coexisting ligamentous instabilities or untreated damage will disrupt articular biomechanics and therefore negatively impact the effectiveness of any prior intervention with regard to the artificial cartilage (26).

\section{References}

1 Cai X, Lin Y, Ou G, Luo E, Man Y, Yuan Q and Gong P: Ectopic osteogenesis and chondrogenesis of bone marrow stromal stem cells in alginate system. Cell Biol Int 31: 76-783, 2007.

2 Danisovic L, Varga I, Zamborsky R and Bohmer D: The tissue enhineering of articular cartilage: cells, scaffolds and stimulating factors. Exp Biol Med (Maywood) 237: 10-17, 2012.

3 Maehara H, Sotome S, Yoshii T, Torigoe I, Kawasaki Y, Sugata Y, Yuasa M, Hirano M, Mochizuki N, Kikuchi M, Shinomiya K and Okawa A: Repair of Large Osteochondral Defects in Rabbits Using Porous Hydroxyapatite/Collagen (Hap/Col) and Fibroblast Growth Factor -2 (FGF-2). J Orthop Res 28: 677-686, 2010.

4 Mano JF and Reis RL: Osteochondral defects: present situation and tissue engineering approaches. J Tissue Eng Regen Med 1: 261-273, 2007.

5 Mollenhauer JA: Perspectives on articular cartilage biology and osteoarthritis. Injury 39: 5-12, 2008.

6 Archer C and Ralphs J: Regenerative medicine and biomaterials for the repair of connective tissues. Woodhead Publishing Limited, Cambridge, 2010.

7 Haasper C, Zeichen J, Meister R, Krettek C and Jagodzinski M: Tissue engineering of osteochondral constructs in vitro using bioreactors. Injury 39: 66-76, 2008.

8 Kamarul T, Ab-Rahim S, Tumin M, Selvaratnam L and Ahmad TS: Preliminary study of the effects of glucosamine sulphate and Chondroitin sulphate on surgically treated and untreated focal cartilage damage. Eur Cell Mater 21: 259-271, 2011.

9 Shah MR, Kaplan KM, Meislin RJ and Bosco JA: Articular Cartilage Restoration of the Knee. Bull NYU Hosp Jt Dis 65: 51-60, 2007.

10 Bhosale AM and Richardson JR: Articular cartilage: structure, injuries and review of management. Br Med Bull 87: 77-95, 2008.

$11 \mathrm{Ge} \mathrm{Z,} \mathrm{Li} \mathrm{C,} \mathrm{Heng} \mathrm{BC,} \mathrm{Cao} \mathrm{G} \mathrm{and} \mathrm{Yang} \mathrm{Z:} \mathrm{Functional}$ biomaterials for cartilage regeneration. J Biomed Mater Res A 100: 2526-2536, 2012. 
12 Proffen B, McElfresh M, Fleming B and Murray M: A comparative anatomical study of the human knee and six animal species. Knee 19: 393-499, 2012.

13 Hunziker EB: Articular cartilage repair: basic science and clinical progress. A review of the current status and prospects. Osteoarthritis and Cartilage 10: 432-463, 2002.

14 Shah RN, Shah NA, Del Rosario MM, Hsieh C, Nuber G and Stupp SI: Supramolecular design of self-assembling nanofibers for cartilage regeneration. Proc Natl Acad Sci USA 107: 3293 3298, 2010.

15 Chu CR, Szczodry M and Bruno S: Animal Models for Cartilage Regeneration and Repair. Tissue Eng Part B Rev 16: 105-115, 2010.

16 Reinholz GG, Lu L, Saris DBF, Yaszemski MJ and O'Driscoll SW: Animal models for cartilage reconstruction. Biomaterials 25: 1511-1521, 2004.

17 Widuchowski W, Tomaszewski W, Widuchowski J and Czamara A: Contemporary possibilities of treatment of cartilage lesions with special regard to the knee joint. Ortop Traumatol Rehab 13: 327-341, 2011.

18 Fox SM: Multimodal management of canine osteoartritis. CRC Press, Taylor\&Francis Group, 2nd edition, Boca Raton, 23-150, 2017.

19 Zeyland J, Lipiński D, Juzwa W, Pławski A and Słomski R: Structure and application of select glycosaminoglycans. Med Wet 62: 139-144, 2006.

20 Hildner F, Albrecht C, Gabriel C, Redl H and Van Griensven M: State of the art and future perspectives of articular cartilage regeneration: a focus on adipose-derived stem cells and plateletderived products. J Tissue Eng Regen Med 5: 36-51, 2011.

21 Pochwalski M, Urbanowska E and Wojtowicz A: Tissue engineering. Aplication in dentistry. Platelet rich plasma. Part I. Nowa Stomatologia 1-2: 17-22, 2000.

22 Johnson SA and Tobias KM: Platelet-Rich Plsma and Autologus Conditioned sera. In: Veterinary Surgery Small Animal 2nd edition, Elsevier Inc., St. Louis Missouri, pp. 40-48, 2018.

23 Baltzer AWA, Moser C, Jansen SA and Krauspe R: Autologous conditioned serum (Orthokine) is an effective treatment for knee osteoarthritis. Osteoarthritis and Cartilage 17: 152-160, 2009.

24 Schepsis AA and Busconi BD: Sports Medicine; Articular cartilage injuries (chapter 30) Pylawka TK, Kang RW, Cole BJ. Philadelphia: Lippincott Williams \& Wilkins, ISBN: 9780781756532, 418-429, 2006.

25 Steinwachs MR, Guggi TH and Kreuz PC: Marrow stimulation techniques. Injury Suppl 1, 1: 26-31, 2008.

26 Farr J, Cole B, Dhawan A, Kercher J and Sherman S: Clinical Cartilage Restoration. Evolution and Overview. Clin Orthop Relat Res 469: 2696-2705, 2011.

27 Kalson NS, Gikas PD, Briggs TWR: Current strategies for knee cartilage repair. Int J Clin Pract 64: 1444-1452, 2010.

28 Knutsen G, Drogset JO, Engebretsen L, Grontvedt T, Ludvigsen TC, Loken S, Solheim E, Strand $\mathrm{T}$ and Johansen O: A randomized multicenter trial comparing autologous chondrocyte implantation with microfracture: Long-term follow-up at 14 to 15 years. J Bone Joint Surg Am 98: 1332-1339, 2016.

29 Tyyni A and Karlsson J: Biological treatment of joint cartilage damage. Review article. Scand J Med Sci in Sports 10: 249-265, 2000.

30 Chiang $\mathrm{H}$ and Jiang CC: Repair of articular cartilage defects: Review and perspectives. J Formos Med Assoc 108: 87-99, 2009.
31 Fitzpatrick N, Yeadon R and Smith T: Early Clinical Experience with Osteochondral Autograft Transfer for Treatment of Osteochondritis Dissecans of the Medial Humeral Condyle in Dogs. Vet Surg 38: 246-260, 2009.

32 Martowicz M and Laska J: Polymer biomaterials for skin regeneration: a review. Engineering of Biomaterials 13: 2-9, 2010.

33 Bodo G, Hangody L, Modis L and Hurtig M: Autologous osteochondral grafting (mosaic arthroplasty) for treatment of subchondral cystic lesions in the equine stifle and fetlock joints. Vet Surg 33: 588-596, 2004.

34 Gross AE: Repair of cartilage defects in the knee. J Knee Surg 15: 167-169, 2002.

35 Czitrom A, Lancer F, McKee N and Gross A: Bone and cartilage allotransplantation: a review of 14 years of research and clinical studies. Clin Orthop Relat Res 208: 141-145, 1986.

36 Pelttari K, Steck E and Richter W: The use of mesenchymal stem cells for chindrogenesis. Injury 39: 58-65, 2008.

37 Mistry H, Connock M, Pink J, Shyangdan D, Clar C, Royle P, Court R, Biant LC, Metcalfe A and Waugh N: Autologous chondrocyte implantation in the knee: systematic review and economic evaluation. Health Technol Assess 21: 291-294, 2017.

38 Negrin LL and Vécsei V: Do meta-analyses reveal timedependent differences between the clinical outcomes achieved by microfracture and autologous chondrocyte implantation in the treatment of cartilage defects of the knee? J Orthop Sci 18: 940948, 2013.

39 Nixon AJ, Sparks HD, Begum L, McDonough S, Scimeca MS, Moran N and Matthews GL: Matrix-Induced Autologous Chondrocyte Implantation (MACI) Using a Cell-Seeded Collagen Membrane Improves Cartilage Healing in the Equine Model. J Bone Joint Surg Am 99: 1987-1998, 2017.

40 Arakaki K, Kitamura N, Fujiki H, Kurokawa T, Iwamoto M, Ueno M, Kanaya F, Osada Y, Gong JP and Yasuda K: Artificial cartilage made from a novel double-network hydrogel: In vivo effects on the normal cartilage and ex vivo evaluation of the friction property. J Biomed Mater Res A 93: 1160-1168, 2010.

41 Swieszkowski W, Tuan BH, Kurzydlowski KJ and Hutmacher DW: Repair and regeneration of osteochondral defects in the articular joint. Biomol Eng 24: 489-495, 2007.

42 Trzeciak T and Richter M: Biomaterials in articular cartilage lesions repair. Chir Narzadow Ruchu i Ort Pol 73: 107-111, 2008.

43 Jezierska-Woźniak K, Nosarzewska D, Tutas A, Mikołajczyk A, Okliński $\mathrm{M}$ and Jurkowski MK: Use of adipose tissue as a source of mesenchymal stem cells. Postepy Hig Med Dosw 64: 326-332, 2010.

44 Kmiecik B, Skotny-Krakowian A and Rybak Z: A short overview of stem cells. Biomed Eng 21: 40-45, 2015.

45 Kriston-Pál E, Czibula A, Gyuris Z, Balka G, Seregi A, Sükösd F, Süth M, Kiss- Tóth E, Haracska L, Uher F and Monostori E: Characterization and therapeutic application of canine adipose mesenchymal stem cells to treat elbow osteoarthritis. Can J Vet Res 81: 73-78, 2017.

46 Guercio A, Di Marco P, Casella S, Cannella V, Russotto L, Purpari G, Di Bella S and Piccione G: Production of canine mesenchymal stem cells from adipose tissue and their application in dogs with chronic osteoarthritis of the humeroradial joints. Cell Biol Int 36: 189-194, 2012. 
47 Arokoski JPA, Jurvelin JS, Vaatainen U and Helminem HJ: Normal and pathological adaptations of articular cartilage to joint loading. Scand J Med Sci Sports 10: 186-198, 2000.

48 Bouffi C, Djouad F, Mathieu M, Noel D and Jorgensen C: Multipotent mesenchymal stromal cells and rheumatoid arthritis: risk or benefit? Rheumatology 48: 1185-1189, 2009.

49 Gimble JM, Katz AJ and Bunnell BA: Adipose-derived stem cells for regenerative medicine. Circ Res 100: 1249-1260, 2007.

50 Guest DJ, Smith MR and Allen WR: Equine embryonic stemlike cells and mesenchymal stromal cells have different survival rates and migration patterns following their injection into damaged superficial digital flexor tendon. Equine Vet J 42: 636$642,2010$.

51 Nowacki J, Dobrzański LA and Gustavo F: Intramedullary implants for osteosynthesis of long. Open Access Library 11: 52$150,2012$.

52 Nowacka M: Biomaterials for tissue engineering and regenerative medicine. Wiadomosci chemiczne 66: 909-933, 2012.

53 Grad S, Kupsik L, Górna K, Gogolewski S and Alini M: The use of biodegradable polyurethane scaffolds for cartilage tissue engineering: potential and limitations. Biomaterials 24: 5163 $5171,2003$.

54 Grande DA, Halberstadt C, Schwarz R and Manji R: Evaluation of matrix scaffolds for tissue engineering of articular cartilage grafts. J Biomed Mater Res 34: 211-220, 1997.

55 Trzeciak T, Richter M, Suchorska W, Augustyniak E, Lach M, Kaczmarek M and Kaczmarczyk J: Application of cell and biomaterial-based tissue engineering methods in the treatment of cartilage, menisci and ligaments injuries. Int Orthop 40: 615624,2016
56 Stoop R: Smart biomaterials for tissue engineering of cartilage. Injury Suppl 1, 39: 77-87, 2008.

57 Stodolak E, Frączek-Szczypta A and Błażewicz M: Polymer-base nanocomposite for medical application. Composites 10: 322-327, 2010.

58 Xue D, Zheng Q, Zong C, Li Q, Qian S, Zhang B, Yu L and Pan Z: Osteochondral repair using porous poly(lactide-co-glycolide)/ nano-hydroxyapatite hybrid scaffolds with undifferentiated mesenchymal stem cells in a rat model. J Biomed Mater Res A 94: 259-270, 2010.

59 Barone DTJ, Raquez JM and Dubois Ph: Bone-guided regeneration: from inert biomaterials to bioactive polymer (nano) composites. Polym Adv Technol 22: 463-475, 2011.

60 Cucchiarini $\mathrm{M}$ and Madry H: Gene therapy for cartilage defects. J Gene Med 7: 1495-1509, 2005.

61 Madry H, Orth P and Cucchiarini M: Gene therapy for cartilage repair. Cartilage 2: 201-205, 2011.

62 Trippel S, Cucchiarini M, Madry H, Shi S and Wang C: Gene therapy for articular cartilage repair. Proc Inst Mech Eng H 221: 451-459, 2007.

63. Trippel SB, Ghivizzani SC and Nixon AJ: Gene-based approaches for the repair of articular cartilage. Gene Ther 11: 351-359, 2004
Received July 16, 2018

Revised August 6, 2018

Accepted August 9, 2018 